\title{
On the Application of Two-Dimensional Chirplets for Resilient Digital Image Watermarking
}

\author{
Jonathan Blackledge \\ Technological University Dublin, jonathan.blackledge@tudublin.ie \\ Oleksandr lakovenko \\ Odessa National Polytechnic University, Ukraine
}

Follow this and additional works at: https://arrow.tudublin.ie/engscheleart

Part of the Electrical and Computer Engineering Commons

\section{Recommended Citation \\ Blackledge, J., lakovenko, 0.:On the Application of Two-Dimensional Chirplets for Resilient Digital Image Watermarking. Accepted for ISSC 2013, Letterkenny Institute of Technology, Letterkenny, Co. Donegal, Ireland, 20-21 June, 2013, p.1-6}

This Conference Paper is brought to you for free and open access by the School of Electrical and Electronic Engineering at ARROW@TU Dublin. It has been accepted for inclusion in Conference papers by an authorized administrator of ARROW@TU Dublin. For more information, please contact arrow.admin@tudublin.ie, aisling.coyne@tudublin.ie,gerard.connolly@tudublin.ie.

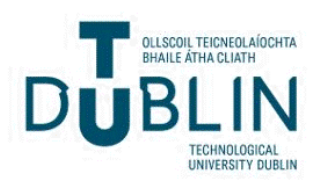




\title{
On the Application of Two-dimensional Chirplets for Resilient Digital Image Watermarking
}

\author{
Jonathan Blackledge* and Oleksandr Iakovenko**
}

\author{
Information and Communications \\ Security Research Group \\ Dublin Institute of Technology \\ Dublin, Ireland \\ E-mail: *jonathan.blackledge@dit.ie
}

\author{
Department of Information Security \\ Odessa National Polytechnic University \\ Odessa, Ukraine \\ **Iakovenko.0leksandr@gmail.com
}

\begin{abstract}
Chirplets (swept-frequency harmonic signals) find applications in several areas, including real and synthetic aperture radar, (Fresnel) optics and image processing. The robustness of chirplets to extreme (additive) noise make them an ideal choice in the role of embedding patterns for resilient digital signal and image watermarking. In this paper we present a new watermarking technique which is based on embedding twodimensional chirplet coded binary sequences in digital images. This approach provides a pattern embedded recovery algorithm which is robust to a variety of distortions. However, chirplets have certain parameters that need to be specified, and, given a set of target characteristics, some particular chirplet parameters can be considered optimal. This paper reports on the optimisation of both the chirplet sets and their associated parameters focusing on a classification of their resilience to image distortion.
\end{abstract}

Keywords - Information hiding, chirplets, robust digital watermarking, correlation detection, optimisation.

\section{INTRODUCTION}

The applications of digital watermarking and information hiding are well known and involve numerous approaches based on both direct data modification (e.g. Least Significant Bit embedding) and transformation based modification using the Discrete Cosine and Wavelet transforms, for example. Methods of encoding the hidden information are also diverse and range from bit-for-bit encryption schemes to the use of Stochastic Diffusion [1][4]. With regard to bit stream encoding for embedding information in digital signals, 'Chirp Coding' [5]-[7] is the one of the most robust techniques with regard to distortion through additive noise. While this technique has been successfully applied to audio signal authentication and self-authentication problems [8] for Digital Rights Management in the audio post-production industry, its application to image watermarking, has, to date, not been investigated. Thus, in this paper we investigate the use of two-dimensional chirplets for embedding binary data in digital images, which, to the best of the author's knowledge, is an original contribution to the field. After briefly reviewing what chirplets are and the combinations available in Section II, Section III studies their optimisation with regard to Bit-Error-Rates. On the basis of this study, new watermark embedding and extraction algorithms are presented in Sections IV and V, respectively, with a focus on the robustness of the approach to image distortion as given in Section VI.

\section{Chirplet Combinations}

Chirplets can be defined in a number of related but strictly different forms, and, by combining these forms, different chirp coding methods can be developed. This is a central theme of the work reported in this paper and in this section we introduced the one-dimensional and two-dimensional chirplet combintions that are available. 


\section{a) One-dimensional Chirplets}

One-dimensional chirps are swept-frequency cosinusoidal signals. In a linear chirp, for example, the frequency $f$ of oscillation is a linear function of time $t$, i.e. $f(t)=f_{0}+k t$ where $f_{0}$ is the carrier frequency and $k$ is a constant that define the extent of the chirp - the 'Chirping Parameter'. 'Chirplets' are time limited chirps or chirps of compact support characterised by a frequency range that sweeps from some minimum to some maximum value or vice versa.

In the time domain, a real chirplet can be defined as (for some arbitrary constant $\theta_{0}$ )

$x\left(t ; f_{0}, k, \theta_{0}\right)=\left\{\begin{array}{l}\sin \\ \cos \end{array}\left[2 \pi\left(f_{0} t+\frac{k}{2} t^{2}+\theta_{0}\right)\right]\right.$

or in complex form, as

$x\left(t ; \omega_{0}, \alpha, \beta\right)=\exp \left[i\left(\omega_{0} t+\alpha t^{2}+\beta\right)\right]$

where $\omega$ is the angular frequency, $\alpha=\pi k$ and $\beta=$ $2 \pi \theta_{0}$

\section{b) Two-dimensional Chirplets}

One-dimensional chirplets can be combined to produce two-dimensional chirplets on a row/column by row/column basis to produce a matrix of essentially one-dimensional chirplets. However, for applications to non-seperable image processing, it is preferable to use two-dimensional patterns instead. This approach yields some significant advantages in the robustness of chirplet watermarking which is a focus of the work presented in this paper as discussed later.

Since the application of the technique focuses on digital images, chirplets are used in discrete form in which they appear as vectors of size $N$ composed of consecutive, uniformly sampled real floating point numbers between -1 and +1 inclusively, i.e.

$$
\mathbf{c} \equiv \mathbf{c}_{i} \in[-1,1] \forall i=1,2, \ldots, N
$$

We consider a $N \times N$ matrix $P$ formed from the dyadic tensor product of the vector $\mathbf{c}$ to produce a two-dimensional chirplet as given by the equation

$$
\begin{aligned}
P=\mathbf{c}^{\mathbf{T}} \mathbf{c} \equiv\left(\begin{array}{c}
c_{1} \\
c_{2} \\
\vdots \\
c_{N}
\end{array}\right)\left(\begin{array}{llll}
c_{1} & c_{2} & \ldots & c_{N}
\end{array}\right) \\
=\left(\begin{array}{cccc}
c_{1} c_{1} & c_{1} c_{2} & \ldots & c_{1} c_{N} \\
c_{2} c_{1} & c_{2} c_{2} & \ldots & c_{2} c_{N} \\
\vdots & \vdots & \ddots & \vdots \\
c_{N} c_{1} & c_{N} c_{2} & \ldots & c_{N} c_{N}
\end{array}\right)
\end{aligned}
$$

However, the chirplet vectors used to construct equation $P$ can be taken in both a 'forward' and 'reverse' order. This produces a total of four distinct two-dimensional chirplet patterns as given below:

$$
\begin{aligned}
& P_{1}=\mathbf{c}^{\mathbf{T}} \mathbf{c} \\
& P_{2}=\operatorname{reverse}(\mathbf{c})^{\mathbf{T}} \mathbf{c} \\
& P_{3}=\mathbf{c}^{\mathbf{T}} \text { reverse }(\mathbf{c}) \\
& P_{4}=\operatorname{reverse}(\mathbf{c})^{\mathbf{T}} \text { reverse }(\mathbf{c})
\end{aligned}
$$

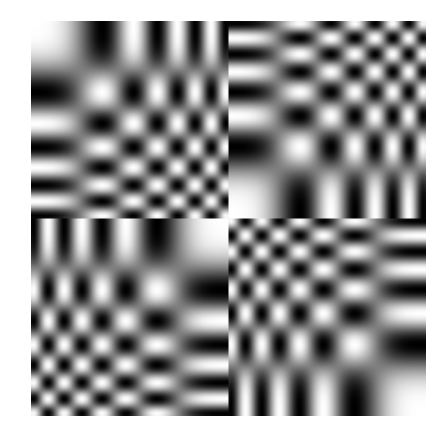

Fig. 1: Four distinct two-dimensional chirplet patterns associated with equations (1)-(4) (clockwise, respectively).

The operation 'reverse' denotes the reversal of the order of the elements in the vector $\mathbf{c}$ used to construct the matrix $P$, i.e. if

$$
\begin{aligned}
\operatorname{reverse}(\mathbf{c} & \left.=\left\{c_{1}, c_{2}, \ldots, c_{N-1}, c_{N}\right\}\right) \\
& =\left\{c_{N}, c_{N-1}, \ldots, c_{2}, c_{1}\right\}
\end{aligned}
$$

The patterns produced by these twodimensional chirplets as given by equations (1)-(4) are shown in Figure 1. Each of these patterns can carry two bits of information: one corresponding to the vertical orientation of the chirp let, and one corresponding to a horizontal orientation.

\section{Optimal Chirplet Combinations and PARAMETERS}

For information hiding applications, a set of embedding patterns is defined to represent different symbols associated with the embedded data. For binary information hiding, the patterns, corresponding to 0 and 1 , should be as separable as possible in order to maximise the robustness of the watermarked host image subject to a range of distortions and perturbations. The embedding patterns for 0 and 1 should be different in some way. For a chirplet signal, the term different can be ambiguous. Thus, if a chirplet $\mathbf{c}$ is considered to be forward, the reverse chirplet is obtained by:

- Value Inversion: -c;

- Time reversal: reverse(c); 
- Time Reversal and Value Inversion: $-\operatorname{reverse}(\mathbf{c})$;

a) Optimal Chirplet Combinations: Robustness to Noise

To illustrate the principle of optimal chirplet combinations, we choose noise robustness as a criterion for chirplet separability for the one-dimensional case. A stream of 3000 random bits $\mathbf{r}$ (i.e. a stream of 1's and 0's) is generated and three sequences of chirplet signals generated, one sequence corresponding to each of the chirplet pairs described above. Thus we generate the signal

$\mathbf{s}=\left\|_{i=1}^{3000} \mathbf{c}_{i} \equiv \mathbf{c}_{1}\right\| \mathbf{c}_{2}\|\cdots\| \mathbf{c}_{3000}$

where

$\mathbf{c}_{i}= \begin{cases}\mathbf{c}_{\text {forward }} & \text { if } \mathbf{r}_{i}=1 \\ \mathbf{c}_{\text {reverse }} & \text { otherwise }\end{cases}$

and $\|$ denote concatenation of the vectors $\mathbf{c}_{i}$ Gaussian noise $\mathbf{n}$ is then added to each sequence where the Noise-to-Signal Ratio (NSR) (the 'Noise Intensity') is taken to be a multiple of the chirplet amplitude. Thus we generate the 'noisy signal'

$\mathbf{s}_{n}=\mathbf{s}+\mathrm{NSR} \mathbf{n}$

the problem now being to recover or 'detect' the bit stream, 'chirp coded' in the vector s. The detection is performed through cross-correlation of the vector $\mathbf{s}_{n}$ with two chirplets:

$\mathbf{d}_{1}=\mathbf{s}_{n} \star \mathbf{c}_{\text {forward }}$

$\mathbf{d}_{2}=\mathbf{s}_{n} \star \mathbf{c}_{\text {reverse }}$

where $\star$ denotes the correlation sum. The correlation maximums are then evaluated to detect each bit in the output stream and by dividing the number of erroneous bits by 3000 , the 'Bit-ErrorRate' (BER) is computed. The computation is repeated with different noise intensities which yield the results shown in Figure 2 The results show that among the chirplet pairs possible, the pair with Time Reversal (the Red Signal given in Figure 2) shows considerably lower BERs for different noise intensities compared to the other chirplet pairs used. The results is effectively the same for different chirplet parameters (e.g. the 'Chirping Parameter'). Thus, in the application of 'Chirp Coding' for hiding binary data in digital signals (e.g. [5], [6] and [7]), Time Reversed Chirplet Pairs provide optimal performance. We now discuss the issue of optimising the chirplet parameters.

\section{b) Optimal Chirplet Parameters}

Chirplets are signals which have following fundamental parameters:

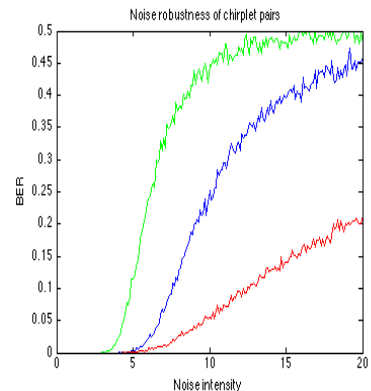

Fig. 2: Bit-Error-Rate (BER) as a function of the Noise Intensity (Noise-to-Signal Ratio) for three different chirplet pairs: Time Reversal (Red), Time Reversal and Value Inversion (Green) and Value Inversion (Blue).

- Starting Frequency $f_{0}$;

- Terminating Frequency $f_{1}$;

- Type of frequency sweep: Linear, Quadric, Logarithmic, etc.

Given the set of target characteristics, some particular chirplets can be considered optimal. Numerical experiments were therefore conducted to define the optimum combination of $\min f_{0}$ and $\operatorname{maxf}_{1}$ for a linearly frequency swept chirplet in terms of achieving maximum robustness to additive Gaussian noise with a Noise Intensity set to 20 . For each combination of $f_{0}$ and $f_{1}>f_{0}$, the value of the BER was computed using 2000 chirplet samples in a sequence using Time Reversed Chirplet Pairs. The resulting surface plot corresponding to the function $\operatorname{BER}\left(f_{0}, f_{1}\right)$ is shown in Figure 3. On

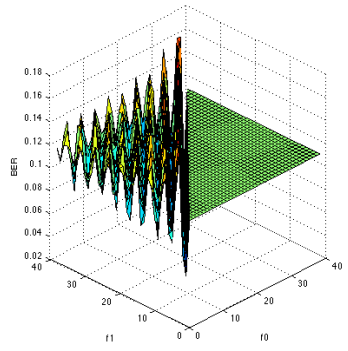

Fig. 3: Bit-Error-Rate (BER) as a function of $f_{0}$ and $f_{1}>f_{0}$ for Time Reversed Chirplet Pairs.

the basis of this surface plot, the optimum chirplet parameters yielding optimal robustness to noise can obtained.

Having established some important numerical issues with regard to using chirplets for signal based bit information hiding, we now consider the details associated with the principal algorithms used for watermarking (grey-level) digital images (with binary information) and assess the robustness of 
the approach to a range of distortions. It is noted that the same approach can be used for full 24bit colour images by decomposing them into their RGB components, details of which lie beyond the scope of this paper.

\section{Watermark Embedding Procedure}

The principal steps associated with the algorithm for embedding binary data in a grey-level image is as follows:

1. Open a Gray-scale Container Image $\mathrm{I}_{c}$, which is taken to be normalised, i.e. $\mathrm{I}_{c} \in[0,1]$ (working with images of size $500 \times 500$ pixels for 'proof of concept' only)

2. Prepare the data for embedding $\mathbf{h}$ which is composed of 25 groups, 2 bits in each group: $\mathbf{h}_{k}=\left\{b_{1}, b_{2}\right\}, k \in[1,25]$.

3. Define the set of four chirplet patterns $\mathrm{P}_{i}, i \in$ $[1,4]$, each chirplet being composed of $100 \times$ 100 elements and where chirplet values lie in interval $[-1,1]$.

4. Generate the Embedding Data Array E be grouping together 25 chirplet patterns $\mathrm{C}_{k}$ into one matrix as given by

$\mathrm{E}=\left[\begin{array}{lllll}\mathrm{C}_{1} & \mathrm{C}_{2} & \mathrm{C}_{3} & \mathrm{C}_{4} & \mathrm{C}_{5} \\ \mathrm{C}_{6} & \mathrm{C}_{7} & \mathrm{C}_{8} & \mathrm{C}_{9} & \mathrm{C}_{10} \\ \mathrm{C}_{11} & \mathrm{C}_{12} & \mathrm{C}_{13} & \mathrm{C}_{14} & \mathrm{C}_{15} \\ \mathrm{C}_{16} & \mathrm{C}_{17} & \mathrm{C}_{18} & \mathrm{C}_{19} & \mathrm{C}_{20} \\ \mathrm{C}_{21} & \mathrm{C}_{22} & \mathrm{C}_{23} & \mathrm{C}_{24} & \mathrm{C}_{25}\end{array}\right]$

where

$\mathrm{C}_{k}= \begin{cases}\mathrm{P}_{1}, & \text { if } \mathbf{h}_{k}=\{0,0\} \\ \mathrm{P}_{2}, & \text { if } \mathbf{h}_{k}=\{0,1\} \\ \mathrm{P}_{3}, & \text { if } \mathbf{h}_{k}=\{1,0\} \\ \mathrm{P}_{4}, & \text { if } \mathbf{h}_{k}=\{1,1\}\end{cases}$

and as shown in Figure 4. Note that the size of $\mathrm{E}$ is equal to the size of $\mathrm{I}_{c}$ but for arbitrary sizes of $\mathrm{I}_{c}$, E can be zero padded.

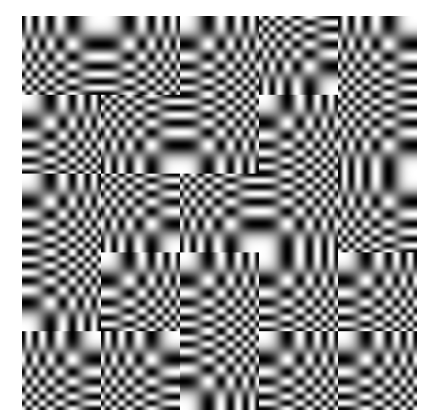

Fig. 4: Embedding map E, consisting of 25 two-dimensional chirplet patterns.
5. The Watermarked image $\mathrm{W}$ is then acquired by addition of the Embedding Image, scaled with an Embedding Rate Factor $R$, to the Container Image, i.e.

$\mathrm{W}=\mathrm{I}_{c}+R \cdot \mathrm{E}$

By changing the value of $R$, it is possible to find an optimal trade-off between invisibility of the watermark and robustness with regard to watermark extraction. Typical values for $R$ lie between 0.01 (maximum invisibility) and 0.07 (maximum robustness).

Embedding can also be accomplished with only one bit per pattern. In this case, only two from four possible two-dimensional chirplet patterns are used. The embedding capacity associated with this approach is half (i.e. 25 instead of 50 bits) but it provides greater robustness and the Embedding Ratio can be set to a lower value.

\section{WATERMARK EXTRACTION}

Chirplet pattern detection is performed in the same way as the matching detection of any pattern, namely, through (two-dimensional) crosscorrelation of the watermarked image with each of four embedding patterns, i.e.

$\mathrm{D}_{i}=\mathrm{W} \star \star \mathrm{P}_{i}, \quad i \in[1,4]$

where $\star \star$ denotes the two-dimensional crosscorrelation sum. As with other matched filtering methods, if a region of the image contains an embedded chirplet pattern which matches the correlation kernel, then the central part of the crosscorrelation surface has a distinct maximum showing the presence of a matching pattern as illustrated in (Figure 5). This property allows us to perform two important operations:

- Make a decision with regard to the embedded pattern based on direct comparison.

- Detect the position of the centre of the embedding block upon extraction of the watermarked channel, e.g. when the watermarked image has been subjected to shift, re-sampling and other deformations and distortions.

If we assume that the original block-by-block positioning is known, then the extraction procedure can be formalised using the following steps:

1. The watermarked image $\mathrm{W}$ undergoes a twodimensional cross-correlation (equation 5) with each of the four embedding patterns used.

2. For each embedding region, only the central components of the correlation surface are 


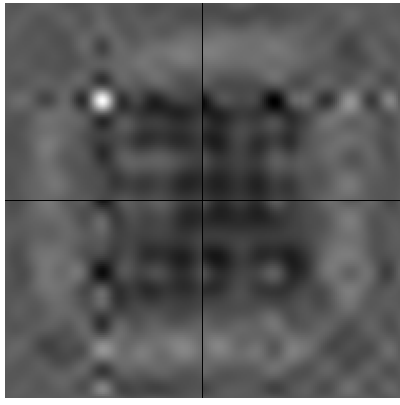

Fig. 5: Results of cross-correlating one region with four chirplet patterns. The bright spot in the centre of the top-left block indicates the presence of the first chirplet pattern in a region.

stored. These 'components' can be defined in a number of ways and a detection filter implemented. In this work, the central component is defined as a square $7 \times 7$ matrix extracted from the centre in each block of the correlation surface.

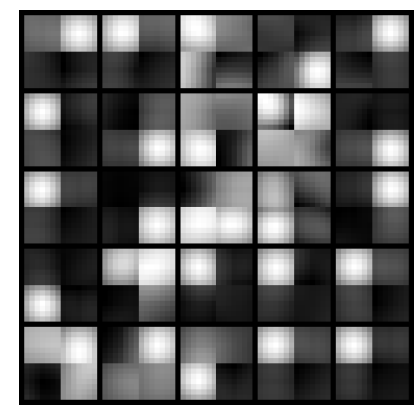

Fig. 6: The central components generated by cross-correlating four times - see (5) - for each embedding region.

3. Compute the average value of the central component in each of the four central regions for each embedding block:

$$
A_{i}=\mathbf{M}\left(\text { Central part of } \mathrm{D}_{i}\right), \quad i \in[1,4]
$$

4. Compare the average values $A_{i}$ and locate the maximum value together with its associated index $i$ which uniquely identifies $\mathbf{h}_{k}$.

Given the basic steps above, we now explore the robustness of the watermarking method when the watermarked image is subjected to a range of distortions.

\section{Robustness TO DISTORTION}

To quantify the practical viability of the proposed method, a number of numerical experiments were conducted with the aim of measuring the actual watermark robustness characteristics given that

\begin{tabular}{|l|c|}
\hline \multicolumn{1}{|c|}{ Type of Distortion } & BER \\
\hline No Distortion & 0.06 \\
\hline Shift (By any Extent) & 0.06 \\
\hline Noise (Intensity) & 0.06 \\
\hline 5 & 0.06 \\
\hline 10 & 0.06 \\
\hline 20 & 0.06 \\
\hline 40 & 0.04 \\
\hline 80 & 0.06 \\
\hline 160 & 0.10 \\
\hline 320 & 0.12 \\
\hline Barrel (Distortion Intensity) \\
\hline 0.5 & 0.20 \\
\hline 1 & 0.06 \\
\hline Gaussian Blur (Blur Radius) \\
\hline 5 & 0.08 \\
\hline 10 & 0.10 \\
\hline 15 & 0.16 \\
\hline 20 & 0.12 \\
\hline Rotate (CCW Degrees) \\
\hline 1 & 0.18 \\
\hline 2 & \\
\hline \multicolumn{2}{|c|}{} \\
\hline
\end{tabular}

Table 1: Summary of results obtained through the numerical experiments undertaken to assess the robustness of the method to image distrortion using the five categories listed, i.e. Shift, Noise, Barrel, Gaussian Blurring and Rotation.

the data watermarking and recovery processes are the same as in algorithms outlined in Sections IV and V. This included the following basic steps:

1. Distorting the watermarked image in some way, the exact types of distortions being listed in Table 1.

2. Extracting a watermark from the distorted image using the watermark extraction algorithm described in $\mathrm{V}$.

3. Comparing the embedded and extracted data and computing the Bit-Error-Rate.

The results are compounded in Table 1 . In some regions of the image, hidden data is extracted with errors even when there is no distortion. The cause of this lies in the nature of the 'container image', some regions of which can contain some pronounced chirplet-like patterns themselves. However, an appropriate selection of chirplet parameters together with an adjustment of the Embedding Rate Factor $R$ can diminish this effect. Any shift in the image pixels can be detected and thereby eliminated and the Noise Intensity is taken as a percentage of the image intensity.

While performing a barrel (pincushion) distortion, a new radius of each point $r_{n}$ is calculated from the old radius $r$ according to the equation 
$r_{n}=r+a r^{3}$, where $a$ is the Barrel Distortion Intensity (divided by $10^{6}$ ). These results are achieved without correction with regard to recovering the initial positions. However, it is noted that this correction can significantly improve robustness to these types of distortion.

\section{Summary AND Conclusion}

In this article we present a new watermarking system for digital images that uses a special coding kernel based on different chirplet forms as a binary data embedding pattern. The unique properties of chirplets (as explored in this work) allow for the achievement of very high rates of robustness to image distortion especially with regard to distortion by noise. The main aim of this work has been to develop a set of numerical experiments to verify the robustness properties of the watermarking method and to define a set of optimal chirplet parameters including best estimates for the combination of minimum and maximum chirplet frequencies and optimal chirplet pairs for embedding binary data. The results show that chirplet pairs with time reversal provides significantly lower BitError-Rates when compared to other pairs. With regard to the application of the method to image watermarking application in general, the method is shown to be adequately robust to Barrell and Rotational distortion (relatively to other image watermarking methods including the application of the Discrete Cosine and Wavelet transforms, for example) but highly robust to Blur and significantly robust to additive noise. In the latter, case, and, to the best of the authors knowledge and research, the method could well prove to be the most resilient of all image watermarking methods developed to date in terms of its robustness to noise. This is in keeping with previous work undertaken on chirp coding [5] including the use of multilevel chirp coding [6] and a further investigation is therefore required into the application chirplets for the two-dimensional multi-channel watermarking of digital image using the methodology and applications discussed [9] and [10].

\section{ACKNOWLEDGMents}

Jonathan Blackledge is supported by the Science Foundation Ireland Stokes Professorship Programme. Oleksandr Iakovenko is funded by the Erasmus Mundus Action II co-operation and mobility programme EWENT (East-West European Network on Higher Technical education) managed by Warsaw University of Technology, Poland. Both authors are grateful to Dr Marek Rebow at Dublin Institute of Technology for arranging the collaborative research programme being undertaken by the authors.

\section{REFERENCES}

[1] J. M. Blackledge and E. Coyle, "e-Fraud Prevention based on the Self-authentication of eDocuments", 4th Int, Conference on Digital Society, IEEE Comp. Society (ISSN: 978-07695-3953-9), pp. 329-338, 2010.

[2] J. M. Blackledge, "Information Hiding using Stochastic Diffusion for the Covert Transmission of Encrypted Images", ISSC2010 UCC Cork, 23-24 June, pp. 1-6, 2010.

[3] J. M. Blackledge and A. R. Al-Rawi, "Application of Stochastic Diffusion for Hiding High Fidelity Encrypted Images", ISAST Trans. On Computing and Intelligent Systems, Vol. 3, No. 1, pp. 24-33, 2011.

[4] J. M. Blackledge and A. R. Al-Rawi, "Steganography using Stochastic Diffusion for the Covert Communication of Digital Images", IANEG Int. J. of Appl. Math., Vol. 4, No. 4, pp. 270-298, 2011.

[5] J. M. Blackledge, "Digital Watermarking and Self-Authentication using Chirp Coding", ISAST Trans. on Elec. \& Sig. Proc., Vol. 1, No. 1, pp. 61-71, 2007.

[6] J. M. Blackledge and O. Farooq, "Audio Data Verification and Authentication using Frequency Modulation based Watermarking", ISAST Trans. on Elec. and Sig. Proc. Vol. 3, No 2, pp. 51-63, 2008.

[7] J. M. Blackledge and E. Coyle, "SelfAuthentication of Audio Signals by Chirp Coding", Proc. DAFx09, Como, Italy 1-4 Sept. 09, Vol. 12, No. 1, pp. 1-8, 2009.

[8] J. M. Blackledge, Self-Authentication of Audio Data for Copyright Protection, Technology to License, Dublin Institute of Technology, July, 2009. http://www.dit. ie/hothouse/technologiestolicence/ availableforlicense/ict/

[9] J. M. Blackledge, A. R. Al-Rawi and R. Hickson, "Multi-Channel Audio Information Hiding" , Proc. DAFx2012, York University, 17-21, September, pp. 309-316, 2012.

[10] J. M. Blackledge and A. R. Al-Rawi, Multi-Channel Digital Rights Management for Audio Post-Production, Technology to License, Dublin Institute of Technology, August, 2012. http://www.dit . ie/hothouse/technologiestolicence/ availableforlicense/ict/ 